\title{
Treating acute cystitis with biodegradable micelle-encapsulated quercetin
}

\author{
This article was published in the following Dove Press journal: \\ International Journal of Nanomedicine \\ 7 May 2012 \\ Number of times this article has been viewed
}

\section{Bi Lan Wang' \\ Xiang Gao ${ }^{1,2}$ \\ Ke Men' \\ Jinfeng Qiu' \\ Bowen Yang ${ }^{3}$ \\ Ma Ling Gou' \\ Mei Juan Huang' \\ Ning Huang ${ }^{2}$ \\ Zhi Yong Qian' \\ Xia Zhao' \\ Yu Quan Wei'}

'State Key Laboratory of Biotherapy and Cancer Center, West China Hospital, West China Medical School, ${ }^{2}$ Department of Pathophysiology, College of Preclinical and Forensic Medical Sciences, ${ }^{3}$ College of Life Science, Sichuan University, Chengdu, People's Republic of China
Correspondence: Ma Ling Gou State Key Laboratory of Biotherapy and Cancer Center, West China Hospital, West China Medical School, Sichuan University, Chengdu 61004I, People's Republic of China

Tel +862885164063

Fax +862885164060

Email basad@।63.com

\begin{abstract}
Intravesical application of an anti-inflammatory drug is an efficient strategy for acute cystitis therapy. Quercetin (QU) is a potent anti-inflammatory agent; however, its poor water solubility restricts its clinical application. In an attempt to improve water solubility of QU, biodegradable monomethoxy poly(ethylene glycol)-poly( -caprolactone) (MPEG-PCL) micelles were used to encapsulate QU by self-assembly methods, creating QU/MPEG-PCL micelles. These QU/MPEG-PCL micelles with DL of 7\% had a mean particle size of $\sim 34 \mathrm{~nm}$, and could release QU for an extended period in vitro. The in vivo study indicated that intravesical application of MPEG-PCL micelles did not induce any toxicity to the bladder, and could efficiently deliver cargo to the bladder. Moreover, the therapeutic efficiency of intravesical administration of QU/MPEG-PCL micelles on acute cystitis was evaluated in vivo. Results indicated that QU/ MPEG-PCL micelle treatment efficiently reduced the edema and inflammatory cell infiltration of the bladder in an Escherichia coli-induced acute cystitis model. These data suggested that MPEG-PCL micelle was a candidate intravesical drug carrier, and QU/MPEG-PCL micelles may have potential application in acute cystitis therapy.
\end{abstract}

Keywords: nanomedicine, MPEG-PCL, self-assembly

\section{Introduction}

Urinary tract infections (UTIs) are one of the most common bacterial infections, and Escherichia coli bacteria are the main cause of UTI. ${ }^{1-4}$ Bacterial infection of the bladder always leads to acute cystitis. Patients with acute cystitis account for $95 \%$ of all visits to physicians for UTIs. Commonly, patients with acute cystitis have symptoms of dysuria and increased frequency and urgency of urination, which greatly affect their quality of life..$^{5}$ In the past decades, great efforts have been made to develop efficient therapeutics to treat acute cystitis. Despite many patients with acute cystitis having benefited from these efforts, ${ }^{6}$ acute cystitis therapy still poses great challenges.

Currently, acute cystitis is commonly treated by systemic application of antibiotics and anti-inflammation agents. However, only a small amount of systemically administered drugs can reach the bladder. Intravesical administration means directly instilling the drug solution into the bladder through a urethral catheter, ensuring maximum delivery of active ingredients to the bladder. ${ }^{7-10}$ In addition, intravesical drug administration has other potential benefits such as avoiding the first-pass metabolism. Compared with systemic drug administration, intravesical drug administration, as a local drug delivery method, can increase drug utilization and reduce systemic toxicity and side effects. Thus, intravesical application of therapeutics attracts some attention for cystitis therapy. 
Quercetin (QU; 3,3,4,5,7-pentahydroxyflavone) is one of the most abundant flavonoids found in plants with biological effects; its molecular structure is presented in Figure 1A. ${ }^{11,12}$ To the authors' knowledge, QU has a wide range of biological properties, including anti-viral, ${ }^{13}$ anti-inflammatory, ${ }^{14}$ anti-oxidative, ${ }^{15}$ and anti-tumor activities. ${ }^{16,17}$ In recent years, the anti-inflammatory effect of QU has been well recognized, ${ }^{18,19}$ suggesting that QU as an anti-inflammatory drug has promising clinical application. Recently, it was found that QU can be used to prevent interstitial cystitis. ${ }^{20}$ The authors presumed that QU might have potential application in intravesical therapy of acute cystitis, and set out to prove this hypothesis in this work. However, QU is water insoluble, and developing an aqueous formulation for QU remains difficult, greatly restricting the clinical application of QU. Therefore, it is necessary to develop an aqueous QU formulation as well as study the application of QU in depth in vivo.

Nanotechnology provides a novel platform for delivery of lipophilic drugs. ${ }^{21,22}$ Encapsulation of hydrophobic drugs into nanoparticles can render the drug completely dispersible in solution. Biodegradable polymeric nanoparticles are regarded as excellent candidates for drug-delivery systems. ${ }^{23-25} \operatorname{Poly}(\varepsilon-$ caprolactone) (PCL)/poly(ethylene glycol) (PEG) copolymers are biodegradable, amphiphilic, and easy to produce, showing promising applications in drug-delivery systems. ${ }^{26,27}$ Monomethoxy PEG (MPEG)-PCL is a diblock PCL/PEG copolymer; its molecular structure is shown in Figure 1B. The authors have previously used MPEG-PCL micelles to deliver curcumin, creating a novel curcumin formulation with potential application in colon cancer therapy. ${ }^{28} \mathrm{In}$ an attempt to develop an aqueous formulation for QU, MPEG-PCL micelles were used to encapsulate QU, creating QU/MPEGPCL micelles. The effect of intravesical application of QU/ MPEG-PCL micelles on acute cystitis was then evaluated. These results suggest that QU/MPEG-PCL micelles are a new formulation of QU, with potential applications in bacterial cystitis therapy.

A

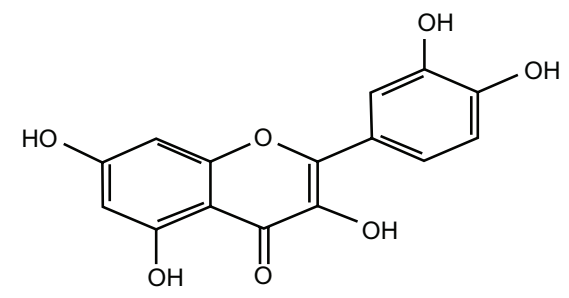

\section{Materials and methods}

\section{Materials}

Escherichia coli DH5 $\alpha$ was saved in the laboratory and was cultured at $37^{\circ} \mathrm{C}$ on solid Luria-Bertani (LB) medium or in liquid LB medium. QU was purchased from Sigma-Aldrich (St Louis, MO). MPEG-PCL diblock copolymer with a designed molecular weight of 4000 was synthesized according to previous reports..$^{27,28}$

Female BALB/c mice (8-10 weeks old) were purchased from the Laboratory Animal Center of Sichuan University (Chengdu, China). All studies involving mice were approved by the Institute's Animal Care and Use Committee.

\section{Preparation of QU/MPEG-PCL micelles}

QU/MPEG-PCL micelles were prepared by a self-assembly method. Briefly, QU (7 mg) and MPEG-PCL diblock copolymer $(93 \mathrm{mg}$ ) were co-dissolved in $6 \mathrm{~mL}$ of organic solvent (dichloromethane $[\mathrm{DCM}]:$ methanol $=2: 1$ ), followed by evaporation under reduced pressure in a rotary evaporator at $55^{\circ} \mathrm{C}$. Normal saline (NS) $(5 \mathrm{~mL})$ was then added into the polymer and drug mixture, allowing the self-assembly of MPEG-PCL and QU, creating core-shell structured QU/MPEG-PCL micelles with core-encapsulated QU. The QU/MPEGPCL micelle solution was filtered using a syringe filter (pore size: $220 \mathrm{~nm}$ ) (Millex-LG, Millipore Co, Billerica, MA) to remove the impurity and bacteria. Finally, the prepared QU/MPEG-PCL micelles were lyophilized and stored at $4^{\circ} \mathrm{C}$.

\section{Characterization of QU/MPEG-PCL micelles}

The particle size and zeta potential of QU/MPEG-PCL micelles were determined by dynamic light scattering (DLS) (Malvern Nano-ZS 90; Malvern Instruments, Malvern, UK). The temperature was kept at $25^{\circ} \mathrm{C}$ during the measuring process. All results were the mean of three test runs.

B

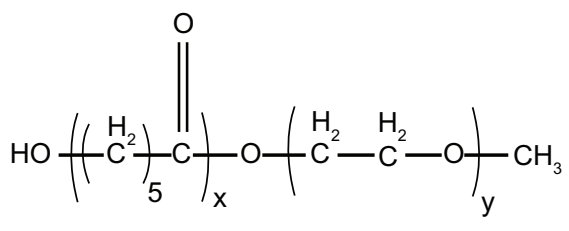

Figure I Molecular structure of (A) quercetin and (B) monomethoxy poly(ethylene glycol)-poly( $\varepsilon$-caprolactone) copolymer. 
The morphology of QU/MPEG-PCL micelles was observed under a transmission electron microscope (H-6009IV; Hitachi, Tokyo, Japan): micelles were diluted with distilled water and placed on a copper grid covered with nitrocellulose. Samples were negatively stained with phosphotungstic acid and dried at room temperature.

The concentration of QU was determined by highperformance liquid chromatography (HPLC) (Waters Alliance 2695; Waters, Milford, MA). The solvent delivery system was equipped with a column heater and a plus autosampler. Detection was carried out on a Waters 2996 detector. Chromatographic separations were performed on a reversed phase C1 8 column $(4.6 \times 150 \mathrm{~mm}-5 \mu \mathrm{m}$, Sunfire Analysis column $)$, with the column temperature kept at $35^{\circ} \mathrm{C}$. Methanol-water $(70 / 30, \mathrm{v} / \mathrm{v})$ was used as eluent at a flow rate of $1 \mathrm{~mL} \cdot \mathrm{min}^{-1}$.

Drug loading (DL) and encapsulation efficiency (EE) of QU/MPEG-PCL micelles were determined as follows: Briefly, $10 \mathrm{mg}$ of lyophilized QU/MPEG-PCL micelles were dissolved in $0.1 \mathrm{~mL}$ DCM and diluted with methanol. The amount of QU in the solution was determined by HPLC. Finally, the DL and EE of QU/MPEG-PCL micelles were calculated according to equations (1) and (2):

$$
\begin{gathered}
\mathrm{DL}=\frac{\text { Drug }}{\text { polymer }+ \text { Drug }} \times 100 \% \\
\mathrm{EE}=\frac{\text { Experimental drug loading }}{\text { Theoretical drug loading }} \times 100 \%
\end{gathered}
$$

\section{In vitro drug release}

To determine the release kinetics of QU from QU/MPEG-PCL micelles, $0.5 \mathrm{~mL}$ of QU/MPEG-PCL micelle solution was placed in a dialysis bag (molecular weight cutoff, $3.5 \mathrm{kDa}$ ). Dialysis bags were incubated in $30 \mathrm{~mL}$ of phosphate buffer solution (pH 7.4) containing Tween ${ }^{\circledR} 80$ (Sigma-Aldrich) $(0.5 \% \mathrm{w} / \mathrm{w})$ at $37^{\circ} \mathrm{C}$ with gentle shaking. At predetermined time points, the incubation medium was replaced with fresh incubation medium. The amount of released drug in the incubation medium was quantified by determining absorbance at $370 \mathrm{~nm}$ using HPLC. This study was repeated three times, and the result was expressed as mean value \pm standard deviation (SD).

\section{Analysis of the acute toxicity of MPEG-PCL micelles}

Fifteen female BALB/c mice ( 8 weeks old) were used to evaluate the acute toxicity of MPEG-PCL micelles after intravesical application. These mice were divided into three groups (five mice per group): one group as the control did not receive any treatment, while the other two groups were treated with NS or MPEG-PCL micelles $(50 \mathrm{mg} / \mathrm{mL} \times 0.1 \mathrm{~mL}$ ), respectively. Twenty-four hours after perfusion, all mice were killed by cervical vertebra dislocation. Bladders were immediately removed, weighted, and fixed in neutral buffer formalin (10\%). The bladders were then paraffin-embedded, and sections were stained with hematoxylin and eosin (H\&E). Moreover, the heart, liver, spleen, kidney, and lung of each mouse were also examined by $\mathrm{H} \& \mathrm{E}$ staining.

\section{Distribution in vivo}

Thirty-three female BALB/c mice ( 8 weeks old) were used to evaluate the distribution of MPEG-PCL micelles. These mice were divided into eleven groups (three mice per group).

Due to its specific fluorescence spectrum, coumarin-6 was used as a model drug to evaluate the capacity of MPEGPCL micelles to deliver cargo to bladder. Coumarin-6/ MPEG-PCL micelles were prepared by a rotary evaporation method. Briefly, coumarin-6 (0.5 mg) and MPEG-PCL diblock copolymer $(99.5 \mathrm{mg}$ ) were co-dissolved in $6 \mathrm{~mL}$ of DCM and evaporated to dryness under reduced pressure in a rotary evaporator. The dried films were hydrated in $5 \mathrm{~mL}$ of $0.9 \%$ NS.

The effect of the administration method on the bladder drug deposition of MPEG-PCL micelle-encapsulated drugs was studied in vivo. Coumarin-6/MPEG-PCL micelles $(0.1 \mathrm{~mL})$ were intravenously or intravesically administered into 8-week-old female BALB/c mice. At different time points $(0.5,1,2,4$, and 6 hours $)$ after administration, mice were killed by cervical vertebra dislocation, and their bladders were immediately harvested and examined by the curmarin-6-associated green fluorescence under a fluorescence light box (Lightools Research, Encinitas, CA). Moreover, to study the effect of administration method on the distribution of MPEG-PCL micelle-encapsulated drugs in vivo, the heart, liver, spleen, lung, and kidney of the mice were also harvested 2 hours after treatment and examined by the curmarin-6-associated green fluorescence under live image analysis instrument (Lightools Research). These experiments were repeated three times, and representative images were obtained.

\section{Establishment of acute cystitis model}

The acute cystitis model was established by intravesical application of bacterial strain E. coli $\mathrm{DH} 5 \alpha$ into 8-weekold female $\mathrm{BALB} / \mathrm{c}$ mice by transurethral catheterization as previously described. ${ }^{29}$ E. coli $\mathrm{DH} 5 \alpha$ was cultured at $37^{\circ} \mathrm{C}$ in $5 \mathrm{~mL} \mathrm{LB}$ for 18 hours, then centrifuged for 1 minute at 
10,000 rpm, washed with phosphate-buffered saline (PBS) three times and diluted to $2 \times 10^{8} \mathrm{CFU} / \mathrm{mL}$. $50 \mu \mathrm{L}$ of this suspension was used to infect each mouse with inoculums of $\sim 1 \times 10^{7} \mathrm{CFU}$.

\section{Treatment of acute cystitis by QU/MPEG-PCL micelles}

Twenty 8-week-old female BALB/c mice were infected with E. coli $\mathrm{DH} 5 \alpha\left(\sim 1 \times 10^{7} \mathrm{CFU}\right.$ per mouse $)$. Twenty-four hours later, the mice were divided into four groups. One group as the control did not receive any treatment, and the other groups were intravesically treated with NS $(0.9 \%)$, MPEGPCL micelle saline solution $(700 \mu \mathrm{g} / \mathrm{mL} \times 100 \mathrm{uL})$ and QU/ MPEG-PCL micelle saline solution(QU: $50 \mu \mathrm{g} / \mathrm{mL} \times 100 \mathrm{uL}$ ), respectively. After 24 hours of treatment, the mice were killed by cervical vertebra dislocation. The bladder of each mouse was harvested and fixed with $10 \%$ buffered formaldehyde. The bladders were then embedded in paraffin, and at least four cross sections ( $5 \mu \mathrm{m}$ thick) were taken from each bladder, followed by H\&E staining.

\section{Statistical analysis}

Data were expressed as the mean \pm SD. Statistical analysis was performed with one-way analysis of variance using SPSS Statistics (version 19.0.1; IBM Corporation, Somers, NY) software. $P$ values less than 0.05 were considered to be statistically significant.

\section{Results}

\section{Preparation and characterization of Qu/MPEG-PCL micelles}

In an attempt to develop an aqueous formulation for QU, biodegradable MPEG-PCL micelles were used to encapsulate QU. QU/MPEG-PCL micelles were prepared through a self-assembly method, as schematically presented in Figure 2. First, MPEG-PCL copolymer and QU were codissolved in a DCM and methanol mixture. Then, the organic solvent was evaporated under reduced pressure in a rotary evaporator, forming an MPEG-PCL and QU mixture. Finally, NS was added to the mixture, allowing the self-assembly of MPEG-PCL and QU in water. In the structure of MPEG-PCL, PEG is the hydrophilic segment, and PCL is the hydrophobic segment; thus, MPEG-PCL micelles always have a core-shell structure, with a PCL core and a PEG shell. The self-assembly of MPEG-PCL and QU created core-shell QU/MPEG-PCL micelles with core-encapsulated QU.

The QU/MPEG-PCL micelles were characterized in detail. The QU/MPEG-PCL micelles had DL and EE of 6.9\% and $98.1 \%$, respectively. In Figure 3A, the particle size distribution spectrum of freshly prepared QU/MPEG-PCL micelles are presented. It was indicated that QU/MPEG-PCL micelles had a very narrow particle size distribution (polydispersity index $[\mathrm{PDI}]=0.12$ ), with a mean particle size of $34.1 \pm 1.2 \mathrm{~nm}$ (determined by DLS). The zeta potential spectrum of QU/ MPEG-PCL micelles is presented in Figure 3B; QU/MPEGPCL micelles had a zeta potential of $-0.269 \mathrm{mV}$. Moreover, the morphology of QU/MPEG-PCL micelles was studied by transmission electron microscopy (TEM), and the result is shown in Figure 3C. According to the TEM image, QU/MPEGPCL micelles were spherical with a mean diameter of $\sim 23 \mathrm{~nm}$. TEM determined the size of dry particles, while the DLS determined the hydrodynamic diameter of particles in water. Because amphiphilic block polymeric micelles always have a loose structure in water, the particle size determined by DLS was always slightly larger than that determined by TEM.

One of the major purposes of the encapsulation of QU in MPEG-PCL micelles was to make QU completely dispersible in aqueous media. The appearance

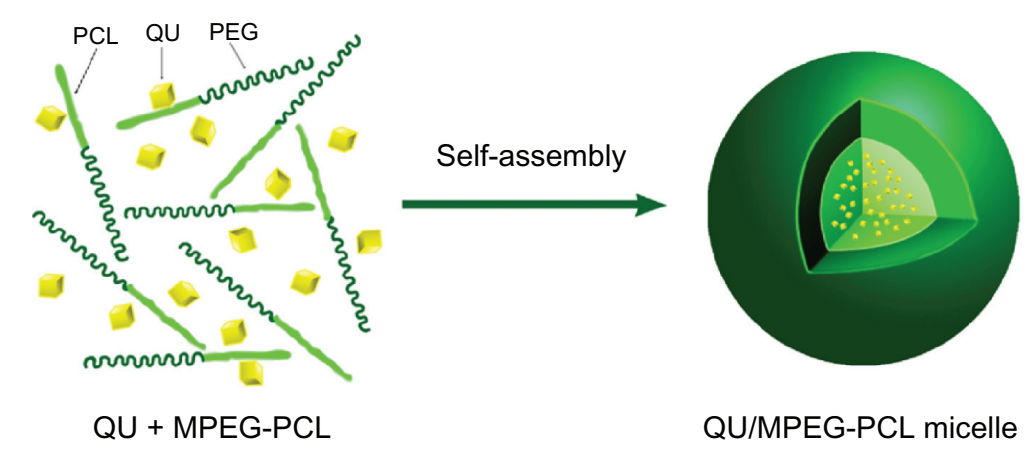

Figure 2 Preparation of QU-loaded MPEG-PCL nanoparticles. MPEG-PCL and QU were co-dissolved in organic solvent, followed by evaporation to dryness under reduced pressure in a rotary evaporator, creating QU and MPEG-PCL mixture (QU + MPEG-PCL).

Notes: The QU and MPEG-PCL mixture was then hydrated in $0.9 \%$ normal saline, allowing QU and MPEG-PCL to self-assemble into QU/MPEG-PCL micelles. This micelle has a core-shell structure (hydrophilic PEG shell and hydrophobic PCL core) with core-encapsulated QU.

Abbreviations: MPEG, monomethoxy poly(ethylene glycol); PCL, poly(e-caprolactone); QU, quercetin. 

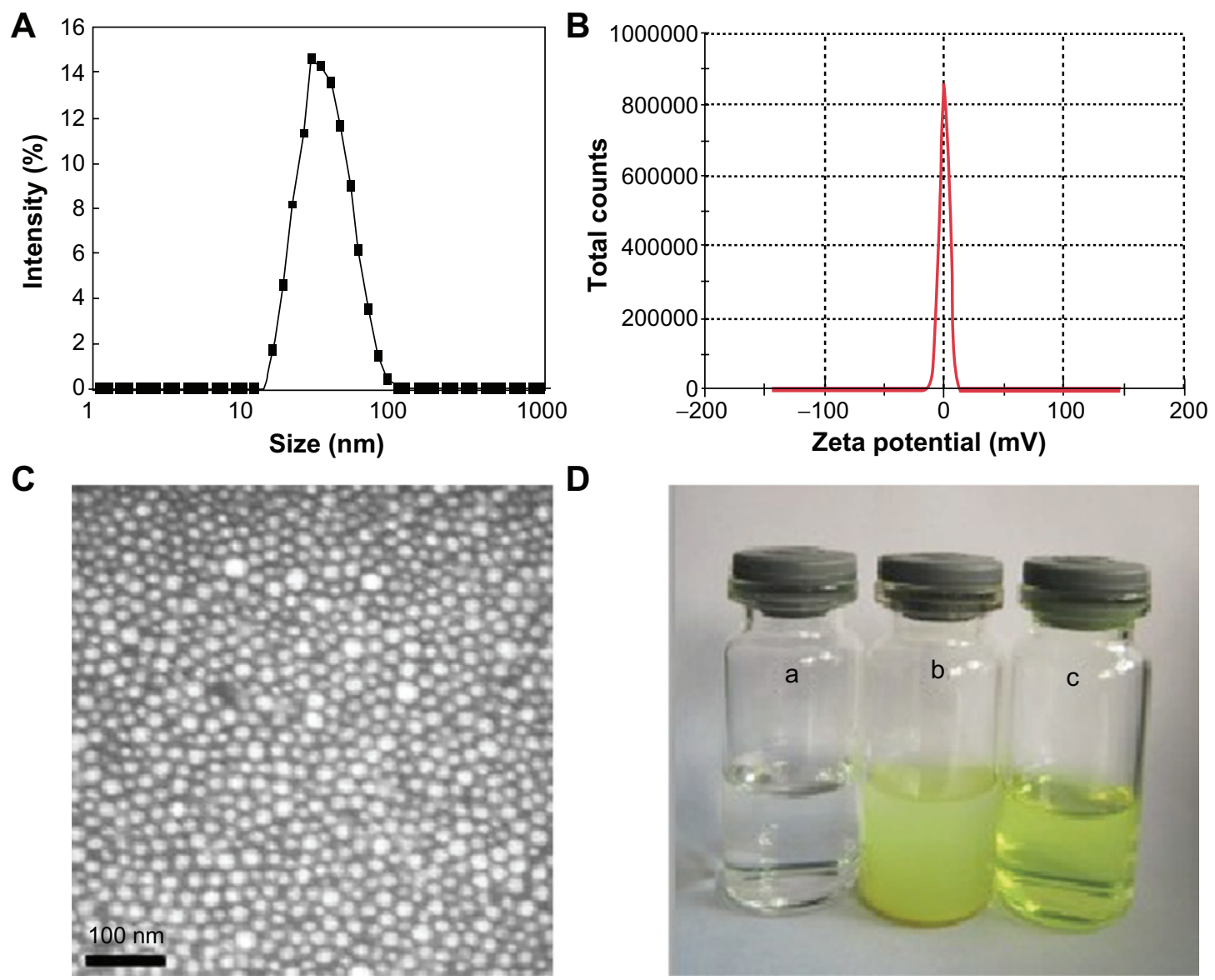

D

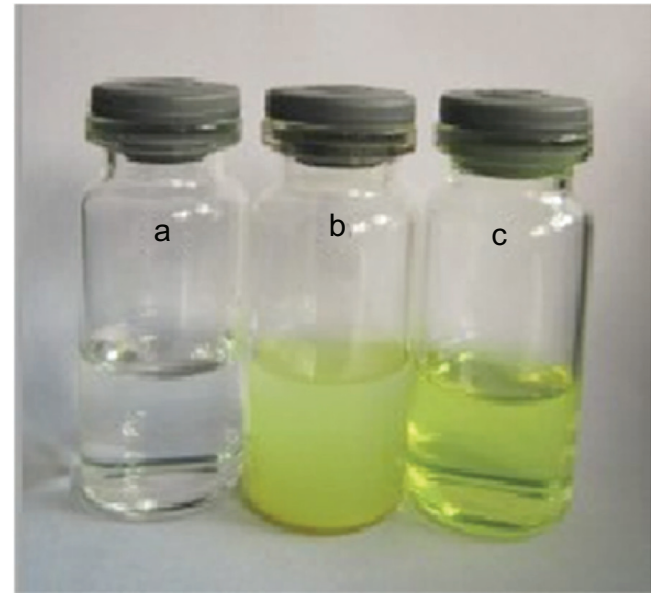

Figure 3 Characterization of QU/MPEG-PCL micelles: (A) size distribution spectrum of QU/MPEG-PCL micelles; (B) zeta potential spectrum of MPEG-PCL micelles; (C) transmission electron microscopy image of QU/MPEG-PCL micelles; (D) the encapsulation of QU in MPEG-PCL/QU nanoparticles renders QU completely dispersible in aqueous media (a, water $(\mathrm{pH}=7.0)$; b, QU in water $(\mathrm{pH}=7.0,2 \mathrm{mg} / \mathrm{mL}) ; \mathrm{c}$, QU/MPEG-PCL micelles in water $(\mathrm{pH}=7.0,2 \mathrm{mg} / \mathrm{mL})$.

Abbreviations: MPEG, monomethoxy poly(ethylene glycol); PCL, poly( $\varepsilon$-caprolactone); $Q U$, quercetin.

of QU/MPEG-PCL micelle aqueous solution is shown in Figure 3D. QU cannot be dissolved in pure water, as confirmed by the observation of a turbid yellow slurry. In contrast, QU/MPEG-PCL micelle solution with an equivalent quantity of QU was transparent, indicating full dispersibility of QU in water.

The release profile of QU/MPEG-PCL micelles in vitro was studied using a dialysis method. As shown in Figure 4, QU was released from QU/MPEG-PCL micelles over an extended period.

\section{Analysis of toxicity of MPEG-PCL micelles}

In this work, the authors attempted to treat acute cystitis by intravesical application of MPEG-PCL micelle-encapsulated QU. Thus, the toxicity of intravesically applied MPEG-PCL micelles was evaluated before treatment experiments. As shown in Figure 5A, intravesical application of MPEGPCL micelles $(50 \mathrm{mg} / \mathrm{mL} \times 0.1 \mathrm{~mL})$ did not significantly induce bladder weight change (control: $16.604 \pm 0.739 \mathrm{mg}$;
NS: $17.032 \pm 1.846 \mathrm{mg}$; MPEG-PCL: $16.874 \pm 0.780 \mathrm{mg}$ ), which implied that the intravesical application of MPEGPCL micelles did not significantly induce bladder edema. Moreover, pathological section of bladder in each treatment group is shown in Figure 5B. We found that intravesical application of MPEG-PCL micelles did not induce any

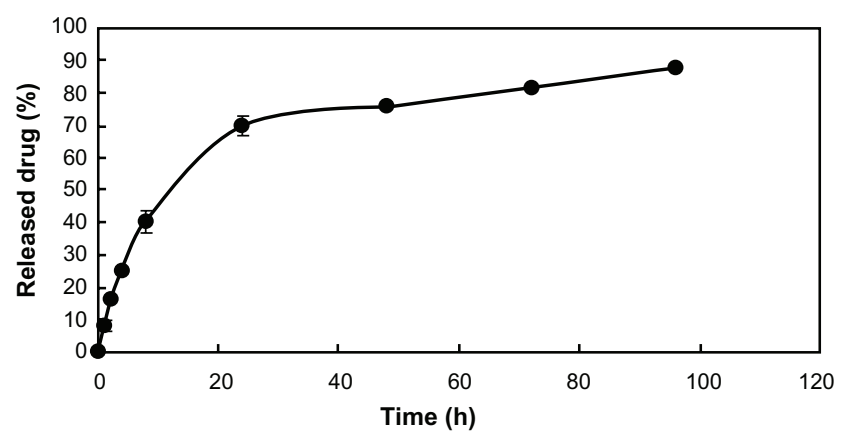

Figure 4 In vitro release study.

Note: The in vitro release profile of QU/MPEG-PCL micelles was examined using a dialysis method.

Abbreviations: MPEG, monomethoxy poly(ethylene glycol); PCL, poly (ع-caprolactone); QU, quercetin. 

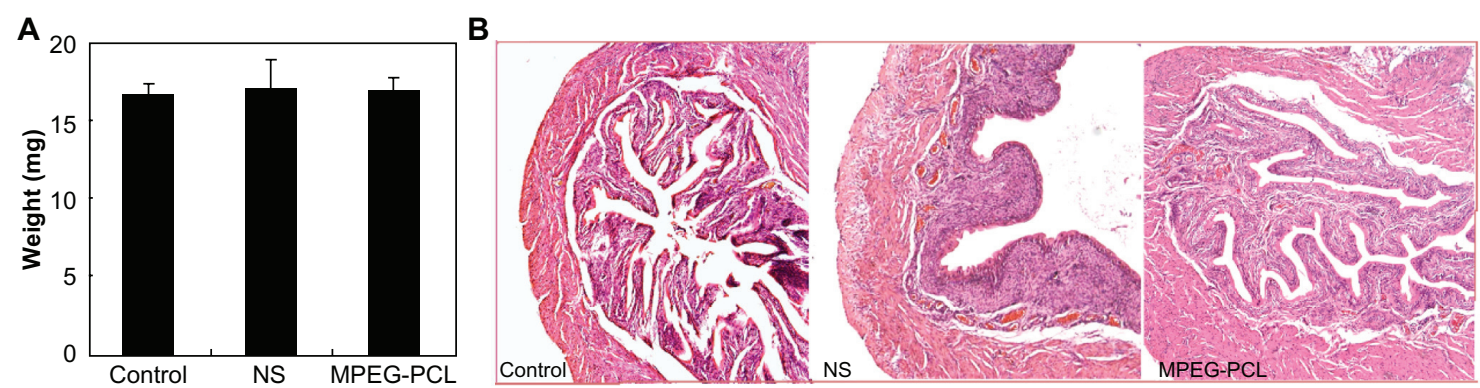

Figure 5 Acute toxicity of intravenously administered MPEG-PCL micelles $(50 \mathrm{mg} / \mathrm{mL} \times 0.1 \mathrm{~mL})$ to bladder. (A) The weight of bladders in different treatment groups $(P>0.05)$; (B) hematoxylin and eosin assay of bladders in different treatment groups.

Note: Little difference was observed among these groups, which indicated that intravesical application of MPEG-PCL had little toxicity to bladder.

Abbreviations: MPEG, monomethoxy poly(ethylene glycol); NS, normal saline; PCL, poly(E-caprolactone).

pathological changes to the bladder. Meanwhile, intravesical application of MPEG-PCL micelles did not cause any toxicity to the heart, liver, spleen, lung, or kidney (data not shown). These results suggest that MPEG-PCL micelles may be used as drug carriers for intravesical drug application.

\section{Distribution in vivo}

The tissue distribution of MPEG-PCL micelle-encapsulated drug after intravenous or intravesical application was studied. Figure 6A shows that green fluorescence appeared in the
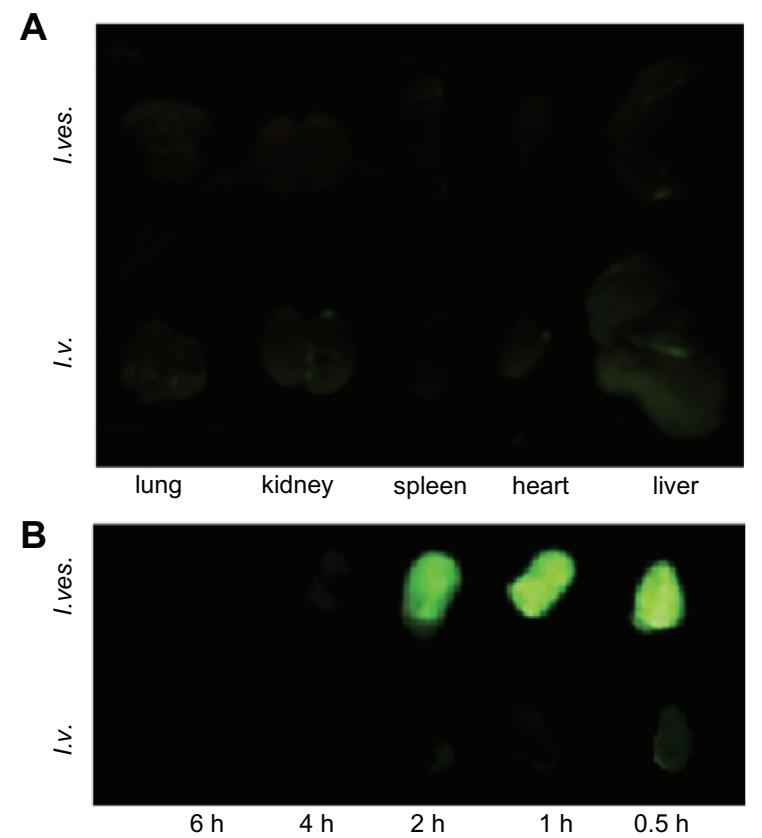

Figure 6 Tissue distribution of MPEG-PCL micelle-delivered cargo after intravenous (I.v.) or intravesical (I.ves.) application. The drug-associated green fluorescence indicated the deposition of drugs. (A) The fluorescence image of organs from mice 2 hours after intravenous or intravesical application of courmarin-6/MPEG-PCL micelles. (B) Fluorescence images of bladders from mice with intravenous or intravesical application of courmarin-6/MPEG-PCL micelles at different time points.

Abbreviations: MPEG, monomethoxy poly(ethylene glycol); PCL, poly( $\varepsilon-$ caprolactone). heart, lung, liver, and kidney 2 hours after intravenous injection, implying drug deposition in these tissues. However, very little green fluorescence appeared in the heart, lung, liver, kidney, and spleen after intravesical administration of courmarin-6/MPEG-PCL, suggesting that little drug can reach these tissues after intravesical administration. Thus, for bladder diseases therapy, intravesical application of therapeutics may cause lower systemic toxicity compared with intravenous application.

The efficiency of MPEG-PCL micelles delivering cargo to bladder was studied after intravenous or intravesical application (see Figure 6B). With intravesical application of courmarin-6/MPEG-PCL micelles, the bladders emanated strong courmarin-6-associated fluorescence, implying the high drug deposition in the bladder. With intravenous application of courmarin-6/MPEG-PCL micelles, the bladders only had slight green fluorescence, suggesting the low efficiency of delivering cargo to bladder. Meanwhile, the residence time of green fluorescence in the bladder with intravesical administration was obviously longer than that with intravenous application. Thus, for bladder diseases therapy, intravesical application of therapeutics may induce higher efficiency of delivering cargos to bladder compared with intravenous application.

\section{Treatment of acute cystitis}

The anti-inflammatory effect of MPEG-PCL micelle-encapsulated QU in the acute cystitis model was assessed. As shown in Figure 7A, the weight of bladders in the QU/MPEG-PCL micelle treatment group was $20.50 \pm 1.44 \mathrm{mg}$, while the weight of bladders in the NS and empty MPEG-PCL micelle treatment group were $43.26 \pm 1.42 \mathrm{mg}$ and $41.42 \pm 1.68 \mathrm{mg}$, respectively. Compared with NS treatment, QU/MPEG-PCL micelle treatment significantly reduced the weight of bladders with severe inflammation $(P<0.05)$, but empty MPEG-PCL 
A

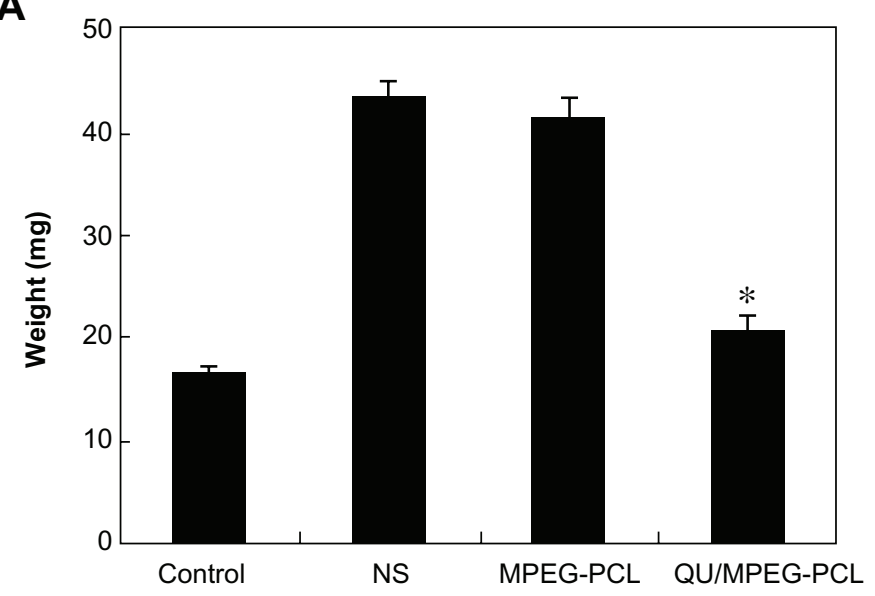

B

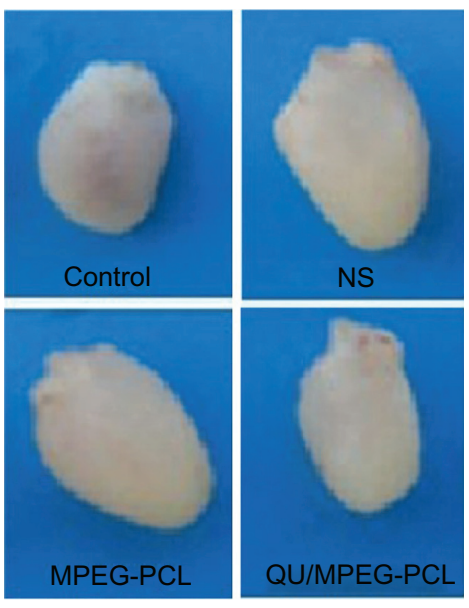

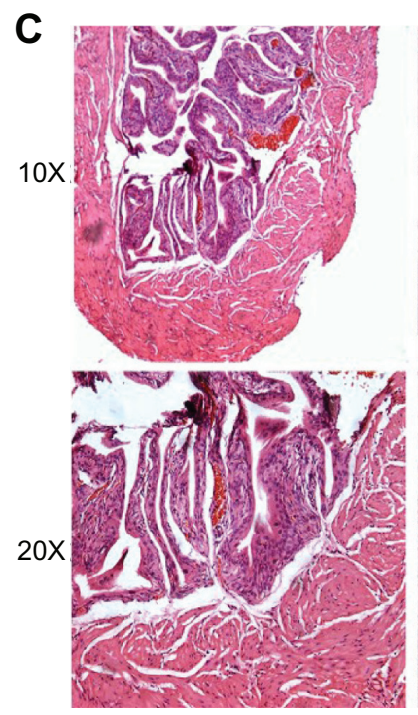

Control

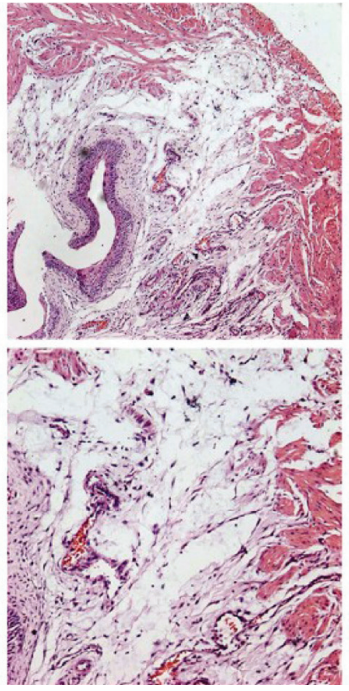

NS

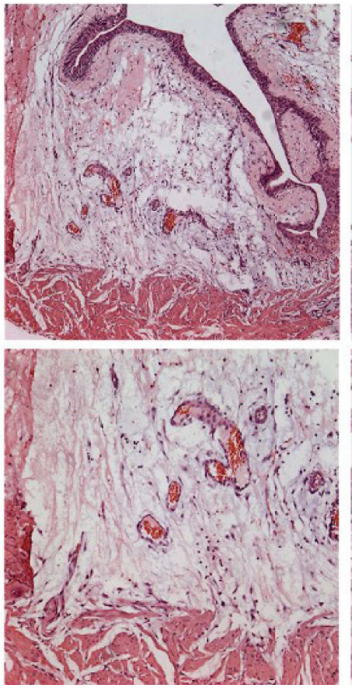

MPEG-PCL

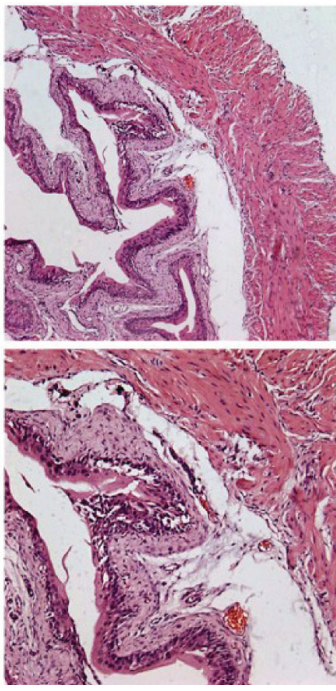

QU/MPEG-PCL

Figure 7 Therapeutic effects of intravesical application of QU/MPEG-PCL micelles on acute cystitis. (A) The weight of bladders in each treatment group. The weight of the bladder in the QU/MPEG-PCL group was less than that in the MPEG-PCL or NS group $(P<0.05)$. (B) Representative images of the bladders in each treatment group. The bladder in the QU/MPEG-PCL group was smaller than that in the NS group, while the bladder in the MPEG-PCL group was as large as that in the NS group. (C) Hematoxylin and eosin assay of the bladders from mice with Control, NS, MPEG-PCL, or Qu/MPEG-PCL treatment.

Notes: Severe edema and lots of inflammatory cell infiltration were observed in the bladders from both the empty MPEG-PCL micelle group and the NS group, indicating that MPEG-PCL had no effect on the treatment of cystitis. However, in the QU/MPEG-PCL treatment group, no obvious edema and inflammatory cell infiltration of the bladder was detected, suggesting that QU/MPEG-PCL micelles have promising application in acute cystitis therapy.

Abbreviations: MPEG, monomethoxy poly(ethylene glycol); NS, normal saline; PCL, poly( $\varepsilon$-caprolactone); QU, quercetin.

micelle treatment did not reduce the weight of bladders with severe inflammation $(P>0.05)$. This indicated that QU-loaded MPEG-PCL micelles effectively inhibited development of acute cystitis compared with NS, while empty MPEG-PCL micelles did not show any anti-inflammatory activity on acute cystitis.

In Figure 7B, the appearance of the bladders in each treatment group is presented. The bladder in the QU/MPEG-PCL group was obviously smaller than that in the NS group, while the bladder in the empty MPEG-PCL micelle group was as large as that in the NS group. This indicated that the edema of the bladder efficiently lessened with QU/MPEG-PCL treatment.
In Figure 7C, the pathological section of bladders in each treatment group is provided. that the figure shows that the bladders in both the empty MPEG-PCL micelle group and the NS group had severe edema and lots of inflammatory cell infiltration. However, in the QU/MPEG-PCL treatment group, the bladder did not have obvious edema and inflammatory cell infiltration. These results suggest that the QU/MPEG-PCL micelles have promising application in acute cystitis therapy.

\section{Discussion}

In this work, QU/MPEG-PCL micelles of uniform size (size, $34.1 \mathrm{~nm}$; PDI, 0.12) and high encapsulation rate (>98\%) 
successfully solved the problem of poor water solubility of QU (Figure 3). These QU/MPEG-PCL micelles could slowly release QU in vitro (Figure 4). Moreover, QU/MPEG-PCL micelles were used to treat acute cystitis, and it was found that intravesical application of MPEG-PCL micelles did not induce any toxicity to the bladder, and intravesical administration of QU/MPEG-PCL micelles efficiently reduced the inflammation of the bladder with $E$. coli-induced acute cystitis.

Patients with acute cystitis always have symptoms of dysuria and increased frequency and urgency of urination, which seriously affect their quality of life. The incidence of acute cystitis is high, and the course of acute cystitis is urgent. If acute cystitis cannot be treated in a timely manner, it will be transformed into chronic cystitis. It can also be transformed into cystitis glandularis, and finally into bladder cancer. It can also induce nephritis. Therefore, timely treatment of acute cystitis is necessary. Acute cystitis therapy has received special attention in the past decades. ${ }^{30}$ The urinary bladder is an ideal organ for regional therapy. The urethra provides easy access of the therapeutic agents to the bladder. Moreover, this current work has proved that intravesical drug application can more efficiently deliver drugs to the bladder, with minimal side effects compared with intravenous application. Therefore, intravesical application of drugs may be an acceptable strategy for acute cystitis therapy. However, this method may have a weakness: intravesical instillation of drugs into the bladder is inconvenient.

Nanotechnology has a wide application in medicine, with the goal of resolving some medical challenges. One of the most important applications of nanotechnology in medicine is using nanoparticles to deliver hydrophobic drugs to create aqueous formulations for these water soluble-poor drugs. QU has great prospects in the treatment of inflammation, ${ }^{31}$ but it is limited in its medical application due to poor water solubility. To address this challenge, the use of liposomes to encapsulate QU has been described. ${ }^{32}$ Recently, MPEG-PCL micelles were used to load curcumin and honokiol, ${ }^{28,33}$ successfully solving their water solubility. In this paper, MPEG-PCL micelles were used to encapsulate QU with the goal of improving the water solubility of QU and creating a novel formulation for QU. Previously, MPEG-PCL micelles had been used to deliver drugs followed by intravenous, subcutaneous, intraperitoneal, or intratumoral application. In this work, MPEG-PCL micelles were used to deliver drugs to the bladder by intravesical application. The results suggested that intravesical application of MPEG-PCL micelle-encapsulated hydrophobic drugs can efficiently deliver cargo to the bladder.
Application of anti-inflammation agents is a commonly used protocol for acute cystitis therapy. QU is a well recognized anti-inflammation agent. ${ }^{31}$ In this paper, QU was used to treat acute cystitis. Results indicated that QU/MPEG-PCL micelle treatment can efficiently reduce the edema and inflammatory cell infiltration of the bladder in an E. coli-induced acute cystitis model. These data proved the hypothesis that QU had potential application in acute cystitis therapy. To the authors' knowledge, this work may be the first attempt to reveal the potential application of QU in acute cystitis therapy.

Overall, nanotechnology provides a novel method for improving the water solubility of QU. Intravesical application of MPEG-PCL micelle-encapsulated QU has potential application in acute cystitis therapy.

\section{Conclusion}

In this paper, the nanoassemblies of QU and MPEG-PCL were studied, creating a novel QU/MPEG-PCL micelle, which is a new and interesting formulation of QU. MPEGPCL micelles may be a safe and efficient intravesical drug-delivery system for hydrophobic drugs. Intravesical administration of MPEG-PCL micelle-encapsulated QU can efficiently reduce the edema and inflammatory cell infiltration of bladders with acute cystitis, showing great potential in acute cystitis therapy.

\section{Acknowledgments}

This work was supported by the Specialized Research Fund for the Doctoral Program of Higher Education (20110181120087), National Key Basic Research Program (973 Program) of China (2011CB910703), National Natural Science Foundation (NSFC30801294) and Huoyingdong Youth Fund from Ministry of Education (No 122030).

\section{Disclosure}

The authors report no conflicts of interest in this work.

\section{References}

1. Foxman B. The epidemiology of urinary tract infection. Nat Rev Urol. 2010;7:653-660.

2. O'Neill LA. Bacteria fight back against Toll-like receptors. Nat Med. 2008;14:370-372.

3. Daniels IR, Zaman SR. Increasing prevalence of antimicrobial resistance among uropathogens. JAMA. 1999;282(4):325-326.

4. Stamm WE, Hooton TM. Management of urinary tract infections in adults. N Engl J Med. 1993;329:1328-1334.

5. Ferry S, Burman LG, Holm SE. Clinical and bacteriological effects of therapy of urinary tract infection in primary health care: relation to in vitro sensitivity testing. Scand J Infect Dis. 1988;20:535-544.

6. Septimus EJ, Kuper KM. Clinical challenges in addressing resistance to antimicrobial drugs in the twenty-first century. Clin Pharmacol Ther. 2009;86:336-339. 
7. Evans RJ. Intravesical therapy for overactive bladder. Curr Urol Rep. 2005;6:429-433.

8. Buyse G, Waldeck K, Verpoorten C, Björk H, Casaer P, Andersson KE. Intravesical oxybutynin for neurogenic bladder dysfunction: less systemic side effects due to reduced first pass metabolism. $J$ Urol. 1998;160:892-896.

9. Walker MC, Masters JR, Parris CN, Hepburn PJ, English PJ. Intravesical chemotherapy: in vitro studies on the relationship between dose and cytotoxicity. Urol Res. 1986;14(3):137-140.

10. Lee H, Cima MJ. An intravesical device for the sustained delivery of lidocaine to the bladder. J Control Release. 2011;149:133-139.

11. Manthey JA. Biological properties of flavonoids pertaining to inflammation. Microcirculation. 2000;7:29-34.

12. Moskaug JØ, Carlsen H, Myhrstad M, Blomhoff R. Molecular imaging of the biological effects of quercetin and quercetin-rich foods. Mech Ageing Dev. 2004;125:315-324.

13. Ohnishi E, Bannai H. Quercetin potentiates TNF-induced antiviral activity. Antiviral Res. 1993;22(4):327-331.

14. Boots AW, Wilms LC, Swennen EL, Kleinjans JC, Bast A, Haenen GR. In vitro and ex vivo anti-inflammatory activity of quercetin in healthy volunteers. Nutrition. 2008;24(7-8):703-710.

15. Miean KH, Mohamed S. Flavonoid (myricetin, quercetin, kaempferol, luteolin, and apigenin) content of edible tropical plants. J Agric Food Chem. 2001;49:3106-3112.

16. Wei YQ, Zhao X, Kariya Y, Fukata H, Teshigawara K, Uchida A. Induction of apoptosis by quercetin: involvement of heat shock protein. Cancer Res. 1994;54:4952-4957.

17. Cheng S, Gao N, Zhang Z, et al. Quercetin induces tumor-selective apoptosis through downregulation of Mcl-1 and activation of Bax. Clin Cancer Res. 2010;16:5679-5691.

18. García-Mediavilla V, Crespo I, Collado PS, et al. The anti-inflammatory flavones quercetin and kaempferol cause inhibition of inducible nitric oxide synthase, cyclooxygenase- 2 and reactive C-protein, and downregulation of the nuclear factor kappaB pathway in Chang Liver cells. Eur J Pharmacol. 2007;557(2-3):221-229.

19. Hämäläinen M, Nieminen R, Vuorela P, Heinonen M, Moilanen E. Anti-inflammatory effects of flavonoids: genistein, kaempferol, quercetin, and daidzein inhibit STAT-1 and NF-B activations, whereas flavone, isorhamnetin, naringenin, and pelargonidin inhibit only NF-B activation along with their inhibitory effect on iNOS expression and NO production in activated macrophages. Mediators Inflamm. 2007;2007:45673.

20. Katske F, Shoskes DA, Sender M, Poliakin R, Gagliano K, Rajfer J. Treatment of interstitial cystitis with a quercetin supplement. Tech Urol. 2001;7(1):44-46.
21. Zhang L, Chan JM, Gu FX, et al. self-assembled lipid-polymer hybrid nanoparticles: a robust drug delivery platform. ACS Nano. 2008;2:1696-1702.

22. Peer D, Karp JM, Hong S, Farokhzad OC, Margalit R, Langer R. Nanocarriers as an emerging platform for cancer therapy. Nat Nanotechnol. 2007;2(12):751-760.

23. Shutava TG, Balkundi SS, Vangala P, et al. Layer-by-layer-coated gelatin nanoparticles as a vehicle for delivery of natural polyphenols. ACS Nano. 2009;3:1877-1885.

24. Shieh YA, Yang SJ, Wei MF, Shieh MJ. Aptamer-based tumortargeted drug delivery for photodynamic therapy. ACS Nano. 2010;4:1433-1442.

25. Wang T, He N. Preparation, characterization and applications of lowmolecular-weight alginate-oligochitosan nanocapsules. Nanoscale. 2010;2:230-239.

26. Zhou S, Deng X, Yang H. Biodegradable poly ( $\varepsilon$-caprolactone)poly (ethylene glycol) block copolymers: characterization and their use as drug carriers for a controlled delivery system. Biomaterials. 2003;24:3563-3570.

27. Gou M, Wei X, Men K, et al. PCL/PEG copolymeric nanoparticles: potential nanoplatforms for anticancer agent delivery. Curr Drug Targets. 2011;12:1131-1150.

28. Gou M, Men K, Shi H, et al. Curcumin-loaded biodegradable polymeric micelles for colon cancer therapy in vitro and in vivo. Nanoscale. 2011;3:1558-1567.

29. Mulvey MA, Lopez-Boado YS, Wilson CL, et al. Induction and evasion of host defenses by type 1-piliated uropathogenic Escherichia coli. Science. 1998;282:1494-1497.

30. Flores-Carreras O, Martínez-Espinoza CJ, González-Ruiz MI Experience in the treatment of interstitial cystitis: review of 17 cases. Ginecol Obstet Mex. 2011;79(3):125-130.

31. Overman A, Chuang CC, McIntosh M. Quercetin attenuates inflammation in human macrophages and adipocytes exposed to macrophageconditioned media. Int J Obes (Lond). 2011;35:1165-1172.

32. Yuan ZP, Chen LJ, Fan LY, et al. Liposomal quercetin efficiently suppresses growth of solid tumors in murine models. Clin Cancer Res. 2006;12(10):3193-3199.

33. Gou M, Zheng X, Men K, et al. Self-assembled hydrophobic honokiol loaded MPEG-PCL diblock copolymer micelles. Pharm Res. 2009;26(9):2164-2173.
International Journal of Nanomedicine

\section{Publish your work in this journal}

The International Journal of Nanomedicine is an international, peerreviewed journal focusing on the application of nanotechnology in diagnostics, therapeutics, and drug delivery systems throughout the biomedical field. This journal is indexed on PubMed Central,

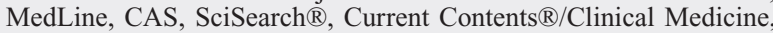

\section{Dovepress}

Journal Citation Reports/Science Edition, EMBase, Scopus and the Elsevier Bibliographic databases. The manuscript management system is completely online and includes a very quick and fair peer-review system, which is all easy to use. Visit http://www.dovepress.com/ testimonials.php to read real quotes from published authors. 